\title{
Screening of Endophytic Fungal Secondary Metabolites from Garcinia kola and Cola nitida for Antioxidant Properties
}

\section{Nwobodo DC ${ }^{1}$, Ihekwereme CP2*, Ugwu MC ${ }^{1}$ and Okoye $\mathrm{FBC}^{3 *}$ \\ 1Department of Pharmaceutical Microbiology and Biotechnology, Faculty of Pharmaceutical Sciences, Nnamdi Azikiwe University, Awka, Anambra State, Nigeria \\ 2Department of Pharmacology and Toxicology, Faculty of Pharmaceutical Sciences,} Nnamdi Azikiwe University, Awka, Anambra State, Nigeria

${ }^{3}$ Department of Pharmaceutical and Medicinal Chemistry, Faculty of Pharmaceutical Sciences, Nnamdi Azikiwe University, Awka, Anambra State, Nigeria

Corresponding authors: Chibueze P Ihekwereme, Department of Pharmacology and Toxicology, Faculty of Pharmaceutical Sciences, Nnamdi Azikiwe University, Awka, Anambra State, Nigeria; Email:

cp.ihekwereme@unizik.edu.ng

Festus B C Okoye, Department of Pharmaceutical and Medicinal Chemistry, Faculty of Pharmaceutical Sciences, Nnamdi Azikiwe University, Awka, Anambra State, Nigeria, Email: fb.okoye@unizik.edu.ng

\section{Abstract}

Objectives: Plant endophytic fungi are novel reservoir of bioactive compounds. The aim of this study was to explore endophytic fungi from Garcinia kola and Cola nitida for antioxidant secondary metabolites.

Methods: Six fungal endophytes, Gn1-3 and Cn1-3 were isolated from the medicinal plants G. kola and C. nitida respectively. The pure endophytes were fermented, their crude secondary metabolites extracted and screened for antioxidant properties using 1, 1-Diphenyl-2-picrylhydrazyl (DPPH) scavenging activities and reducing power (RP) assay. The bioactive principles were identified by dereplication using High Performance Liquid Chromatography-Diode Array Detector (HPLC-DAD) analysis

Results: Extracts of $\mathrm{Cn} 3$ (identified as Trichophyton sp.) and Gc3 (identified as Collectotrichum sp.) demonstrated the most potent antioxidant activity. In the DPPH scavenging assay, extracts of $\mathrm{Cn} 3$ and Gc3 showed an IC 50 of 50.7 and $69.1 \mu \mathrm{g} / \mathrm{ml}$ respectively compared to ascorbic acid $(12.2 \mu \mathrm{g} / \mathrm{ml})$. Extracts of $\mathrm{Cn} 3$, Gc3and positive control (ascorbic acid, $250 \mu \mathrm{g} / \mathrm{ml}$ ) showed reduction values of $1.112 \pm 0.011,1.075 \pm 0.014$ and $1.203 \pm 0.004$, respectively. There was positive correlation between TPC and results obtained in both assay (DPPH, $\mathrm{R}^{2}=0.7039 ; \mathrm{RP}, \mathrm{R}^{2}=0.9778$ ), thus suggesting phenolics-related antioxidant activity. HPLC-DAD analysis revealed extract of $\mathrm{Cn} 3$ contain protocatechuic acid and phydroxybenzoic acid, while Gn3contain 4-hydroxyphenyl acetic acid and indole-3-acetic acid. These compounds have been previously reported to exhibit potent antioxidant activity. In conclusion, this study reveals that endophytic fungi 


\section{Open Access Journal of Pharmaceutical Research}

from both plants possess potential antioxidant properties. The secondary metabolites present in these endophytic fungi have potential for pharmaceutical and agrochemical applications.

Keywords: Garcinia kola; Cola nitida; Antioxidant; Endophytes; Phenolics; Climate change

\section{Introduction}

Oxidative stress, which arises from the presence of reactive oxygen species (ROS) has been established to be involved in the development of various health disorders [1]. Disorders linked to excessive generation of ROS in the human body include cancer, inflammation, Alzheimer's disease, dementia, cardiovascular diseases and atherosclerosis [2,3]. The consumption of fruits and vegetable known to possess antioxidant activity has proved useful in prevention and amelioration of some of these disease states. Recently, enormous research efforts have been directed towards the development of therapeutic antioxidant compounds from these medicinal plants. One of the end results of the commercialization of successful antioxidant compounds derived from plant source may be large scale harvesting and probably destruction of such plants, which in turn could lead to climate imbalance and environmental disruption [4]. This underscores the need to explore alternative and ecofriendly commercial sources of identified medicinal compounds.

Currently, endophytes are viewed as an outstanding and ecofriendly source of bioactive natural products. Endophytes and endophytic fungi refer to microorganisms which grow intercellularly and asymptomatically within living tissues establishing mutual relationship with the host plant [5]. This group of organisms produces bioactive compounds that may provide defense for the host plant against pathogens, and some of these compounds are useful for novel drug discovery [5]. Most plant species habour one or more endophytic organisms [5].

G. kola and C.nitida plants are reputed to exhibit several potent pharmacological activities such as antioxidant, antibacterial, antifungal, antiviral, and anti-inflammatory properties [6-9]. These plants belonging to the family Guttiferae (G. kola) and Sterculiaceae (C.nitida) are popular in African traditional medicine. In our earlier reports, we have shown that Nigerian medicinal plants are good sources of endophytes that can generate secondary metabolites with proven therapeutic potentials [10-12]. In this study, we investigated the secondary metabolic extracts of endophytic fungi from $G$. kola and $C$. nitida for their antioxidant properties.

\section{Materials and Methods}

\section{Sampling/Plant Collections}

Leaf samples of Cola nitida (PCG/474/A/050) and Garcinia kola (PCG/474/A/051) were collected from mature healthy plants from Nsukka, in Enugu State located in South-Eastern part of Nigeria. They were identified by Mrs. Emezie Anthonia of the Department of Pharmacognosy and Traditional Medicine, Faculty of Pharmaceutical Sciences, Nnamdi Azikiwe University, Awka, Nigeria. Plant materials were brought to the laboratory in sterile bags and processed within a few hours after collection. Samples were also deposited at the herbarium of the Department of Pharmacognosy and Traditional Medicine, Faculty of Pharmaceutical Sciences, Nnamdi Azikiwe University, Awka, Nigeria.

\section{Isolation and Purification of Endophytic Fungi}

Fresh plant materials were used for isolation to reduce the risk of contamination. Plant leaves were surface washed in running tap water to remove dust and debris. After proper washing, leaf samples were cut into small segments (about 1-2 mm) and surface-sterilized. The process of surface sterilization involved sequential washing with the under listed solvents. First was in 70\% ethanol for $2 \mathrm{~min}$, second was $2 \%$ sodium hypochlorite ( $\mathrm{NaOCl}$ ) for 2-3 min. Third was in $70 \%$ ethanol for $2 \mathrm{~min}$, and then double rinsing in sterile distilled water. The samples were surface-dried by blotting on sterile filter paper in a laminar flow [13].

About 4 segments from each plant was selected and placed on each Petri dish containing Malt Extract Agar (MEA) supplemented with Chloramphenicol $500 \mathrm{mg} / \mathrm{L}$. The efficacy of surface sterilization was tested by pressing the sterilized segments on to the surface of MEA medium, and removed immediately. The absence of growth of any fungi on the medium after incubation, confirmed that the surface sterilization procedure was effective [14]. The plates were sealed and incubated at $27 \pm 2^{\circ} \mathrm{C}$. Periodically, fungal growth from the leaf segments were monitored 


\section{Open Access Journal of Pharmaceutical Research}

and hyphal tips from distinct colonies emerging from leaf segments were sub-cultured on fresh MEA plates to obtain pure colonies.

\section{Fermentation and Extraction of Secondary Metabolites}

Pure isolates of the fungi were cultured in rice media for the production of secondary metabolites. Small scale fermentation was carried out primarily to perform bioassay for the detection of active metabolites. Solid state fermentation was carried out in $1000 \mathrm{ml}$ Erlenmeyer flasks containing $100 \mathrm{~g}$ of rice media $(200 \mathrm{ml}$ of water was added to the rice and then autoclaved at $121^{\circ} \mathrm{C}$ at $15 \mathrm{psi}$ for $30 \mathrm{~min}$ ). The flasks were inoculated with $3 \mathrm{~mm}$ diameter agar blocks containing pure fungi endophyte and incubated at $25-27^{\circ} \mathrm{C}$ for 30 days. The culture media and the mycelia were extracted in ethyl acetate and separated by filtration. The organic phase was vacuumconcentrated at $40^{\circ} \mathrm{C}$ under reduced pressure using a rotary evaporator.

\section{Sample Preparation}

A stock solution $(1 \mathrm{mg} / \mathrm{ml})$ of each of the extract was prepared by dissolving each extract in 5\% Tween 80 . From the respective stock solutions, two-fold serial dilutions $(250,125,62.5,31.3,15.6$, and $7.8 \mu \mathrm{g} / \mathrm{ml})$ of each of the extracts were prepared.

\section{DPPH (1, 1-Diphenyl-2-picrylhydrazyl) Free Radical Scavenging Assay}

The measurement of the DPPH radical scavenging activity was performed using a previously described method [15]. The free radical scavenging ability of the samples was determined by measuring the absorbance of DPPH at $517 \mathrm{~nm}$ using a spectrophotometer. Ascorbic acid was used as the standard for the assay, and was prepared using same procedure as the test samples. The experiment was carried out in triplicate for each test sample or standard. The percentage (\%) of DPPH scavenging activity was calculated using the formula below:

Where

$$
\% \text { scavenging }=\frac{\text { Abs of control }- \text { Abs of sample }}{\text { Abs of control }} \times 100
$$

Abs of control $=$ Absorbance of DPPH solution without extract or standard

Abs of sample $=$ Absorbance of DPPH solution with extract or standard

$\mathrm{IC}_{50}$ values, which denote the concentration of test sample required to scavenge $50 \%$ of DPPH free radicals were calculated [16].

\section{Reducing Power (RP) Assay Procedure}

The reducing capacity of the extracts was determined using the potassium ferricyanide reduction method as reported by Oyaizu [17].The experiments were done in triplicates and ascorbic acid was used as the reference standard. An increase in absorbance indicated higher reductive ability.

\section{Total Phenolic Content (TPC) Assay}

The total phenol content of the extracts was evaluated using a modified colorimetric method described by Ghasemi et al. [18]. The values obtained were compared to a standard curve prepared with gallic acid solution (Sigma Chemical). The total phenolic content was expressed as milligrams of gallic acid equivalents (mg GAE).

\section{HPLC Analysis}

HPLC analysis was carried out on the samples with a Dionex P580 HPLC system coupled to a photodiode array detector (UVD340S, DionexSoftron GmbH, Germering, Germany). The HPLC-DAD analysis was carried out to identify the active compounds contained in the respective endophytic fungal extracts. Detection was at 235, 254, 280 and $340 \mathrm{~nm}$. The separation column $(125 \times 4 \mathrm{~mm}$; length $\mathrm{x}$ internal diameter) was prefilled with Eurospher10 C18 (Knauer, Germany), and a linear gradient of nanopure water (adjusted to $\mathrm{pH} 2$ by addition of formic acid) and methanol was used as eluent.

\section{Statistical Analysis}

Statistical Program for Social Sciences (SPSS) version 20 was used for all statistical analysis. Data were expressed as mean \pm standard deviation (SD) for three parallel measurements. One way analysis of variance (ANOVA) was carried out to compare the mean obtained in the DPPH scavenging assay and the ferric reduction power assay. Correlation and linear regression were carried out on the results obtained in the DPPH scavenging assay, reduction power assay and total phenolic content, with the total phenolic content serving as the independent variable. The results obtained were considered significant at $\mathrm{P} \leq 0.05$.

\section{Results}

\section{Identification of Principal Secondary Metabolites}

The chromatograms and structures of the compounds identified from the extracts of Gc3, Gc2 and $\mathrm{Cn} 3$ are 
presented in Figures 1a-c. The compounds indole-3-acetic detected by dereplication.

acid, p-hydroxybenzoic acid and protocatechuic acid were
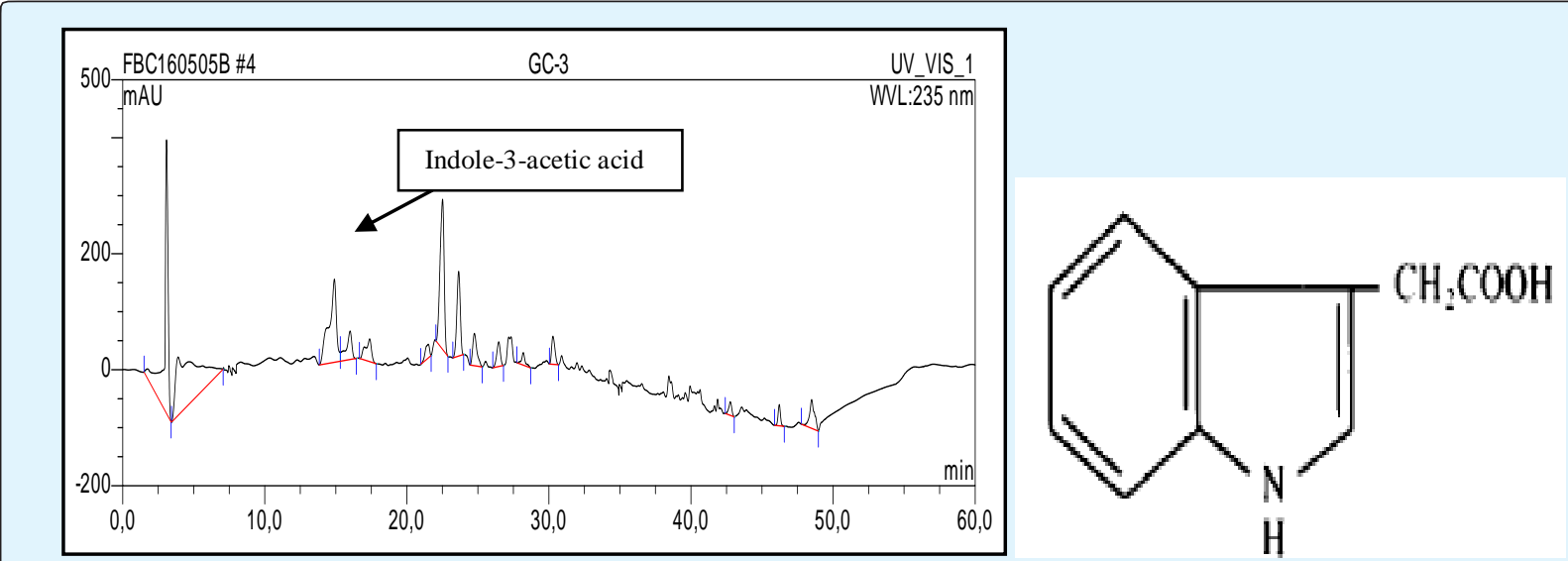

Figure 1a: Chromatogram and structure of Gc3 fungal extract, showing the peak for indole-3-acetic acid.
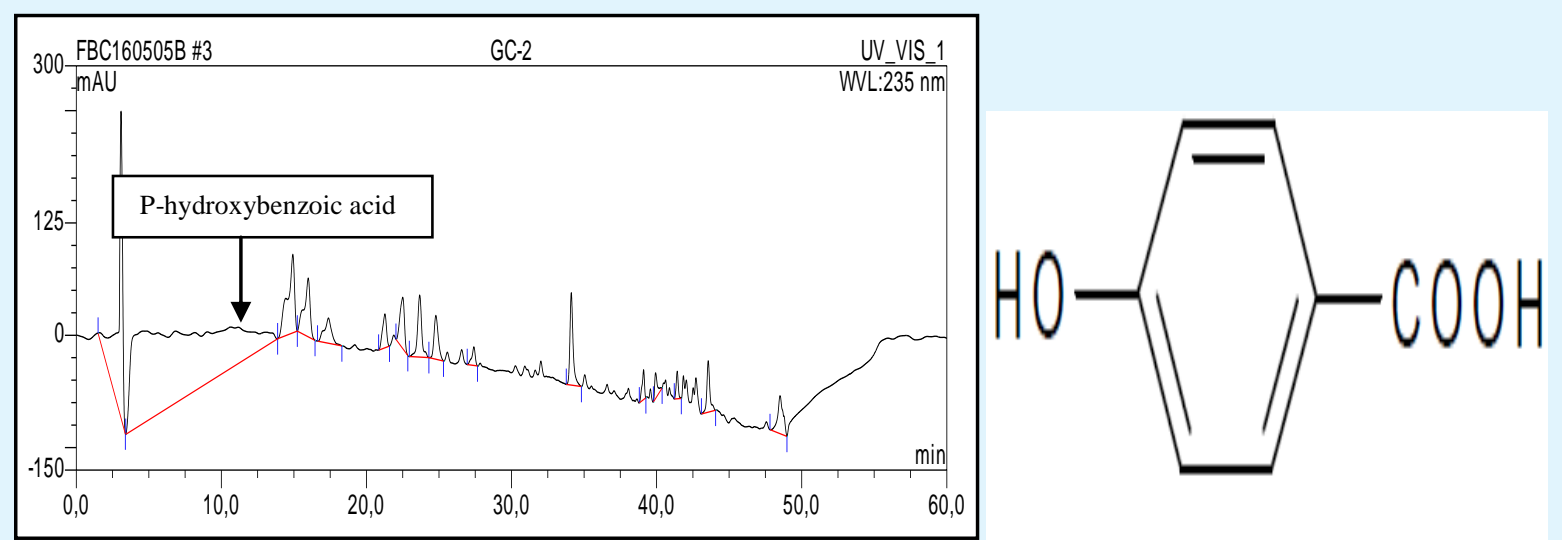

Figure 1b: Chromatogram and structure of Gc2 fungal extract, showing the peak for p-hydroxylbenzoic acid.

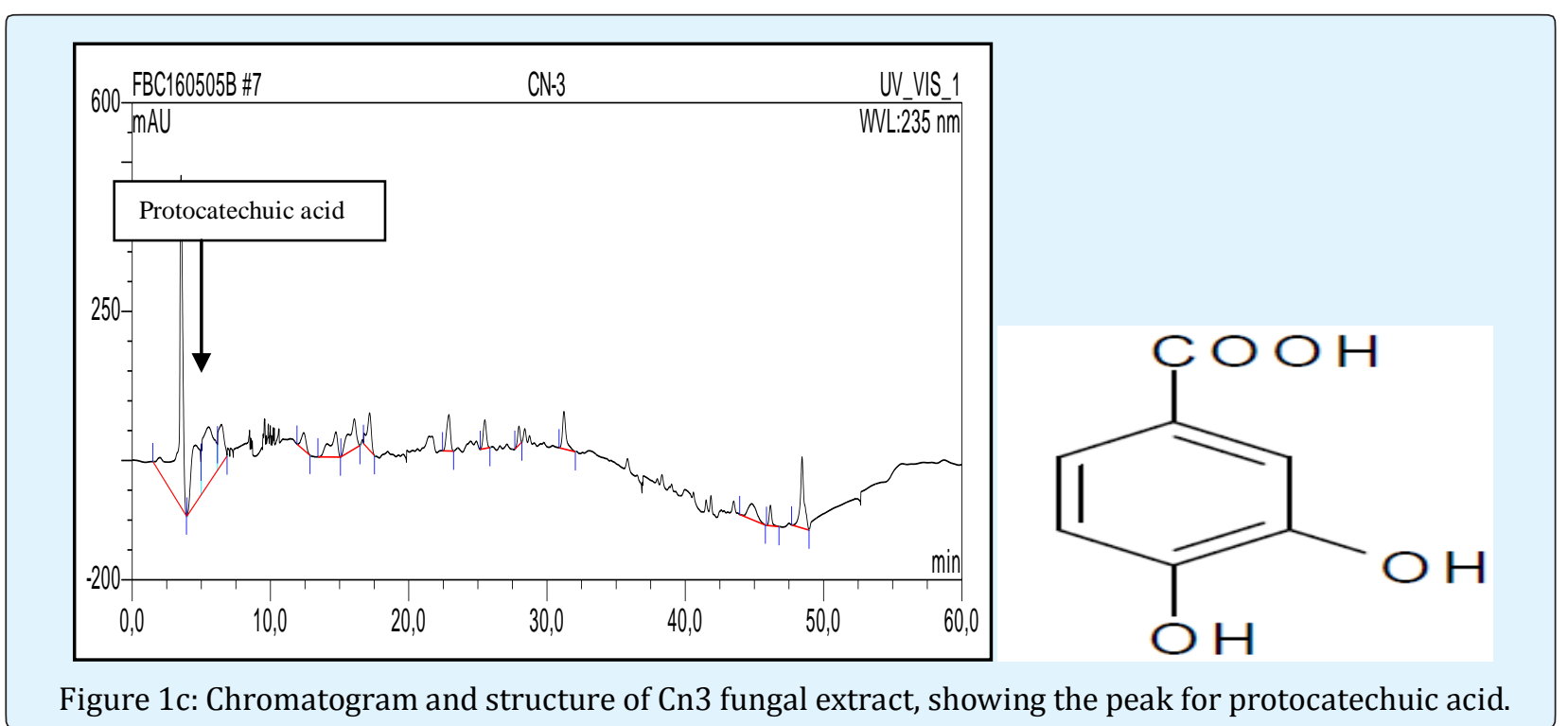

Okoye FBC, et al. Screening of Endophytic Fungal Secondary Metabolites from Garcinia kola and Cola nitida for Antioxidant Properties. J Pharm Res 2017, 1(6):

Copyright $(\subset)$ Okoye FBC, et al. 000136. 


\section{Open Access Journal of Pharmaceutical Research}

\section{DPPH Scavenging Activities of Fungal Endophytic Extracts}

The results of the DPPH scavenging activities and the corresponding $\mathrm{IC}_{50}$ values are shown in (Table 1) and (Figure 2), respectively. All the extracts demonstrated a dose-dependent scavenging activity. The highest percent inhibitions were recorded at $250 \mu \mathrm{g} / \mathrm{ml}$ by $\mathrm{Cn} 3(59.4 \pm$ $1.8)$ and Gc3 $(57.2 \pm 1.8)$. Cn3 showed the lowest $\mathrm{IC}_{50}$ value $(50.7 \mu \mathrm{g} / \mathrm{ml})$, followed by Gc3 $(69.1 \mu \mathrm{g} / \mathrm{ml}), \mathrm{Gc} 4$ $(161.6 \mu \mathrm{g} / \mathrm{ml}), \mathrm{Gc} 1(172.4 \mu \mathrm{g} / \mathrm{ml})$ and $\mathrm{Cn} 2(281.5 \mu \mathrm{g} / \mathrm{ml})$.

\begin{tabular}{|c|c|c|c|c|c|c|}
\hline \multirow{2}{*}{ Extract } & \multicolumn{7}{|c|}{ Concentration $(\boldsymbol{\mu g} / \mathbf{m l})$} \\
\cline { 2 - 7 } & $\mathbf{2 5 0} \boldsymbol{\mu g} / \mathbf{m l}$ & $\mathbf{1 2 5} \boldsymbol{\mu g} / \mathbf{m l}$ & $\mathbf{6 2 . 5} \mathbf{\mu g} / \mathbf{m l}$ & $\mathbf{3 1 . 3} \boldsymbol{\mu g} / \mathbf{m l}$ & $\mathbf{1 5 . 6} \mathbf{\mu g} / \mathbf{m l}$ & $\mathbf{7 . 8} \boldsymbol{\mu g} / \mathbf{m l}$ \\
\hline Gc1 & $53.0 \pm 2.1$ & $48.3 \pm 1.6$ & $40.4 \pm 3.9$ & $37.5 \pm 3.7$ & $34.2 \pm 0.6$ & $32.8 \pm 1.1$ \\
\hline Gc2 & $49.6 \pm 2.3$ & $47.0 \pm 0.0$ & $44.1 \pm 1.4$ & $40.7 \pm 1.6$ & $36.8 \pm 2.2$ & $33.2 \pm 1.4$ \\
\hline Gc3 & $57.2 \pm 1.8$ & $54.0 \pm 1.4$ & $49.4 \pm 1.6$ & $45.6 \pm 1.8$ & $40.9 \pm 2.7$ & $37.0 \pm 1.3$ \\
\hline Gc4 & $51.8 \pm 3.4$ & $49.3 \pm 1.1$ & $44.4 \pm 3.2$ & $41.6 \pm 0.4$ & $36.9 \pm 2.5$ & $32.8 \pm 1.1$ \\
\hline Cn1 & $50.3 \pm 3.6$ & $47.1 \pm 0.2$ & $44.6 \pm 2.4$ & $40.7 \pm 1.1$ & $38.3 \pm 1.1$ & $34.5 \pm 1.4$ \\
\hline Cn2 & $49.2 \pm 1.8$ & $46.9 \pm 0.8$ & $45.0 \pm 1.1$ & $39.4 \pm 1.1$ & $37.8 \pm 0.9$ & $35.6 \pm 1.8$ \\
\hline Cn3 & $59.4 \pm 1.8$ & $54.4 \pm 1.9$ & $51.6 \pm 0.7$ & $48.2 \pm 1.8$ & $42.7 \pm 3.7$ & $39.0 \pm 0.9$ \\
\hline Control & $69.3 \pm 2.1$ & $63.5 \pm 1.6$ & $61.1 \pm 1.3$ & $57.0 \pm 2.3$ & $50.6 \pm 1.9$ & $47.8 \pm 0.4$ \\
\hline
\end{tabular}

Table 1: Percentage (\%) DPPH Scavenging activity.

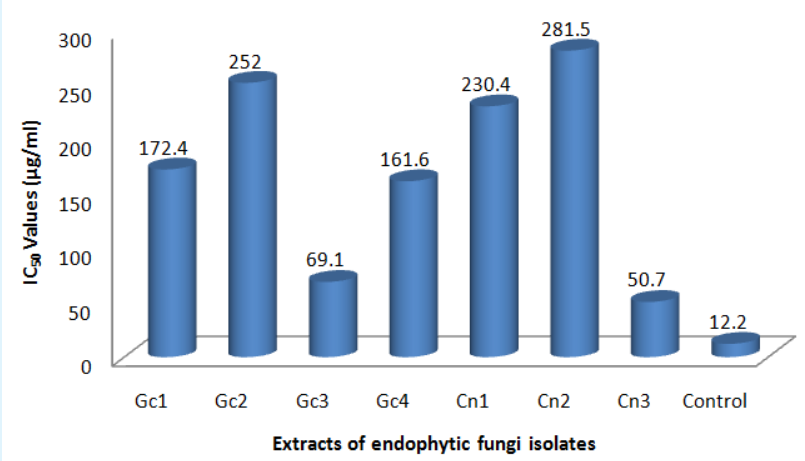

Figure 2: A chart showing the $\mathrm{IC}_{50}$ values of the fungal extracts and control (AA) obtained from the DPPH assay.

\begin{tabular}{|c|c|c|c|c|c|c|}
\hline \multirow{2}{*}{ Extract } & \multicolumn{7}{|c|}{ Concentration of extract $(\boldsymbol{\mu g} / \mathbf{m l})$} \\
\cline { 2 - 7 } & $\mathbf{2 5 0} \mathbf{~} \mathbf{g} / \mathbf{m l}$ & $\mathbf{1 2 5} \mathbf{\mu g} / \mathbf{m l}$ & $\mathbf{6 2 . 5} \mathbf{\mu g} / \mathbf{m l}$ & $\mathbf{3 1 . 3} \mathbf{\mu g} / \mathbf{m l}$ & $\mathbf{1 5 . 6} \mathbf{\mu g} / \mathbf{m l}$ & $\mathbf{7 . 8} \boldsymbol{\mu g} / \mathbf{m l}$ \\
\hline Gc1 & $1.032 \pm 0.006$ & $1.019 \pm 0.010$ & $1.002 \pm 0.010$ & $0.986 \pm 0.007$ & $0.942 \pm 0.027$ & $0.911 \pm 0.007$ \\
\hline Gc3 & $1.075 \pm 0.014$ & $1.032 \pm 0.006$ & $1.019 \pm 0.004$ & $0.993 \pm 0.021$ & $0.971 \pm 0.007$ & $0.912 \pm 0.018$ \\
\hline Gc4 & $1.004 \pm 0.003$ & $0.993 \pm 0.001$ & $0.952 \pm 0.013$ & $0.930 \pm 0.006$ & $0.906 \pm 0.011$ & $0.817 \pm 0.102$ \\
\hline Cn1 & $1.063 \pm 0.014$ & $1.023 \pm 0.010$ & $1.001 \pm 0.013$ & $0.972 \pm 0.000$ & $0.899 \pm 0.030$ & $0.859 \pm 0.003$ \\
\hline Cn2 & $1.031 \pm 0.004$ & $1.020 \pm 0.006$ & $0.990 \pm 0.024$ & $0.973 \pm 0.000$ & $0.956 \pm 0.008$ & $0.816 \pm 0.054$ \\
\hline Cn3 & $1.112 \pm 0.011$ & $1.075 \pm 0.013$ & $1.048 \pm 0.008$ & $1.012 \pm 0.010$ & $0.998 \pm 0.003$ & $0.897 \pm 0.018$ \\
\hline Control & $1.203 \pm 0.004$ & $1.157 \pm 0.016$ & $1.089 \pm 0.010$ & $1.030 \pm 0.014$ & $1.012 \pm 0.003$ & $0.978 \pm 0.016$ \\
\hline
\end{tabular}

Table 2: Mean Absorbance Values for RP Assay.

\section{Determination of Total Phenolic Content (TPC)}

The total phenolic content as well as the variation among the samples analyzed is represented in Figure 3.

\section{Reducing Power (RP) Assay}

The result of the RP (Table 2) show the absorbance value increases as the concentration of each extract increases. Each extract recorded its highest absorbance at $250 \mu \mathrm{g} / \mathrm{ml}$. Cn3 showed the overall highest value of $(1.112 \pm 0.011)$, followed by Gc3 $(1.075 \pm 0.014)$. Both values were lower than the control $(1.203 \pm 0.004)$. 


\section{Open Access Journal of Pharmaceutical Research}

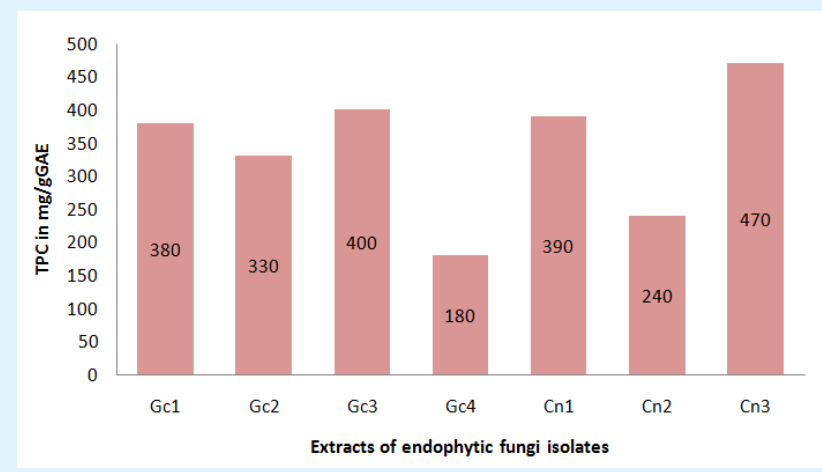

Figure 3: A chart showing an estimate value of the Total Phenol Content (TPC) of isolated fungal extracts.

\section{Discussion}

Radical scavenging activity is very crucial to the survival of living organisms, since their presence results in several damages to the body systems. As earlier said, consumption of antioxidants, either as fruits or vegetables is useful for prevention and amelioration of some health conditions. Interestingly, extracts from fungal isolates of G. Kola and C.nitida demonstrated a marked radical scavenging activity.

Phenolic compounds possess antioxidant properties ${ }^{15}$. The highest scavenging activity was exhibited by $\mathrm{Cn} 3$ and it is interesting to observe that all the compounds identified in $\mathrm{Cn} 3$ are phenolic compounds. The reducing capacities of the extracts of $\mathrm{Cn} 3$ and Gc3 could as well be attributed to the principal compounds identified in the extracts by HPLC analysis. P-hydroxybenzoic acid, protocatechuic acid, and indole-3-acetic acid have all been reported to possess antioxidant effects $[19,20]$.

$\mathrm{IC}_{50}$ values denote the concentration of sample, which is required to scavenge $50 \%$ of DPPH free radicals [16]. From this study, we observed that as the concentration increases, the absorbance value also increases (Table 2). Similarly, using this same assay method, Ayebe, et al. [21] reported the presence of antioxidant compounds in C.nitida. A close look at the overall results of the antioxidant assay from both methods, showed the extracts of the fungi $\mathrm{Cn} 3$ and $\mathrm{Gc} 3$ as the most potent of the test samples. Though phenolic acid and its esters particularly p-hydroxybenzoic acid and protocatechuic acid possess good antioxidant effect [19], indole-3-acetic acid is also reported to scavenge free radicals [20].

Even though DPPH scavenging and reducing powers were based on different mechanisms of antioxidant assay, results from both methods did not show any difference when compared statistically. The similarity observed signifies results from both models are comparable. Furthermore, it provides more reason to believe the extracts truly possess antioxidant activity since it has been suggested that antioxidant activity should not be concluded based on a single test model [22].

It is widely accepted that the antioxidant activity of a plant extract is correlated to its phenolic content [23]. In the present study, strong correlations were observed between phenolic content and antioxidant activity. Statistically, this study showed a positive correlation $\left(\mathrm{R}^{2}=\right.$ 0.978) between results obtained from TPC and RP. Similarly, there was also a strong correlation $\left(\mathrm{R}^{2}=0.704\right)$ between TPC and DPPH scavenging activity. These findings are in line with previous works $[19,24]$ that demonstrated a positive correlation between phenolic content and free radical scavenging activity. In addition, they agree with Ayebe, et al. [21], which attributed the antioxidant activity of $C$. nitida to the presence of phenolic acids. The high phenolic contents of extract from $\mathrm{Cn} 3$ are prime suspects likely to be responsible for the observed antioxidant activity.

As described previously, besides phenolic compounds, other compounds were identified in the fungal extracts, particularly in Gc3 (Table 1). Investigations revealed that those compounds possess different pharmacological properties. Definitely, the pharmacological effects of the fungal extracts are due to the varying phytoconstituents they contain. These observed pharmacological properties could be a consequence of phytoconstituents acting in isolation or in synergy with others. Conversely, the antioxidant activities demonstrated by the fungal extracts in this study can be regarded as moderately potent, when compared to other already known available high natural antioxidants. This may be due to the amount or purity of the actual active compounds in the various fungal extracts.

In conclusion, the results from these in vitro experiments demonstrated that active compounds in the endophytic fungal extracts from $C$. nitida and $G$. kola possess significant antioxidant activities. The secondary metabolites present in these endophytic fungi have potential for pharmaceutical and agrochemical applications. However, large-scale harvesting of these plants could untowardly affect climate change. Alternatively, endophytic fungi from these plant leaves could serve as an eco friendly sustainable source of natural antioxidants for pharmaceutical industries. Furthermore, there may be need to conduct more investigation on these fungal extracts. These would 


\section{Open Access Journal of Pharmaceutical Research}

involve compounds purification and characterization, as well as in vivo research techniques.

\section{References}

1. Bonomini F, Rodella LF, Rezzani R (2015) Metabolic Syndrome, Aging and Involvement of Oxidative Stress. Aging Dis 6(2): 109-120.

2. Umeno A, Biju V, Yoshida Y (2017) In vivo ROS production and use of oxidative stress-derived biomarkers to detect the onset of diseases such as Alzheimer's disease, Parkinson's disease, and diabetes. Free Radic Res 51(4): 413-427.

3. Khurana S, Piche M, Hollingsworth A, Venkataraman K, Tai TC (2013) Oxidative stress and cardiovascular health: therapeutic potential of polyphenols. Can. J Physiol Pharmacol 91(3): 198-212.

4. Lawrence D, Vandecar K (2015) The impact of tropical deforestation on climate and links to agricultural productivity. Nat Publ Gr 5(2): 174.

5. Nisa H, Kamili AN, Nawchoo IA, Shafi S, Shameem N, et al. (2015) Fungal endophytes as prolific source of phytochemicals and other bioactive natural products: A review. Microb Pathog 82: 50-59.

6. Ekene N, Erhirhie OE (2014) Garcinia Kola: a Review of Its Ethnomedicinal, Chemical and Pharmacological Properties. IJCRR 6(11): 1-7.

7. Tcheghebe T, Signe M, Seukep AJ, Tatong FN (2016) Review on traditional uses, phytochemical and pharmacological profiles of Garcinia kola Heckel. Merit Res J Med Med Sci 4(11): 480-489.

8. Joshua PE, Ukegbu CY, Eze CS, Umeh BO, Oparandu LU, et al. (2017) Comparative studies on the possible antioxidant properties of ethanolic seed extracts of Cola nitida (kola nut) and Garcinia kola (bitter kola) on hydrogen peroxide induced oxidative stress in rats. J Med Plants Res 12(22): 367-372.

9. Ashibuogwu MN, Adeosun OI, Akomolafe RO, Sanni DO, Olukiran OS (2016) Diuretic activity and toxicity study of the aqueous extract of Cola nitida seed on markers of renal function and electrolytes in rats. J Complement Integr Med 13(4): 393-404.

10. Okoye FBC, Nworu CS, Akah PA, Esimone CO, Debbab A, et al. (2013) Inhibition of inflammatory mediators and reactive oxygen and nitrogen species by some depsidones and diaryl ether derivatives isolated from Corynespora cassiicola, an endophytic fungus of Gongronema latifolium leaves. Immunopharmacol Immunotoxicol 35(6): 662-668.

11. Okoye FBC, Lu S, Nworu CS, Esimone OC, Proksch P, et al. (2013) Depsidone and diaryl ether derivatives from the fungus Corynespora cassiicola, an endophyte of Gongronema latifolium. Tetrahedron Lett 54(32): 4210-4214.

12. Okoye FBC, Nworu CS, Debbab A, Esimone CO, Proksch P (2015) Two new cytochalasins from an endophytic fungus, KL-1.1 isolated from Psidium guajava leaves. Phytochem Lett 14: 51-55.

13. Arnold AE, Maynard Z, Gilbert GS, Coley PD, Kursar TA (2000) "Are tropical fungal endophytes hyperdiverse?" Ecol Lett 3(4): 267-274.

14. Schulz B, Wanke U, Draeger S, Aust HJ (1993) Endophytes from herbaceous plants and shrubs: effectiveness of surface sterilization methods. Mycol Res 97(12): 1447-1450.

15. Obiagwu MO, Ihekwereme CP, Ajaghaku DL, Okoye FBC (2014) The Useful Medicinal Properties of the Root-Bark Extract of Alstonia boonei (Apocynaceae) May Be Connected to Antioxidant Activity. ISRN Pharmacol 2014: 741478.

16. Santhosh K, Subramanian A, SUJA S, Sudarshan M, Chakraborty A (2013) Free Radical Scavenging Capacity and Antioxidant Activity of an Ayurvedic Medicinal Plant Solanum rubrum Mill. Res J Pharm Biol Chem Sci 4(4): 417-426.

17. Oyaizu M (1986) Studies on products of browning reaction. Antioxidative activities of products of browning reaction prepared from glucosamine. Japanese J Nutr Diet 44(6): 307-315.

18. Ghasemi K, Ghasemi Y, Ebrahimzadeh MA (2009) Antioxidant activity, phenol and flavonoid contents of 13 citrus species peels and tissues. Pak J Pharm Sci 22(3): 277-281.

19. Merkl R, Hradkova I, Filip V, Smidrkal J (2010) Antimicrobial and Antioxidant Properties of Phenolic Acids Alkyl Esters. Czech J Food Sci 28(4): 275-279. 


\section{Open Access Journal of Pharmaceutical Research}

20. Arnao M, Sanchez Bravo J, Acosta M (1996) Indole-3carbinol as a scavenger of free radicals. Biochem Mol Biol Int 39(6): 1125-1134.

21. Ayebe E, Yapi H, Edjeme A, Meite S, Melaine G, et al. (2012) In vivo, in vitro antioxidant activity assessment \& acute toxicity of aqueous extract of Cola nitida (Sterculiaceae). Asian J Biochem Pharm Res 2(4): 144-155.

22. Amudha M, Rani S (2016) Evaluation of In Vitro Antioxidant Potential of Cordia retusa. Indian J Pharm Sci 78(1): 80-86.
23. Piluzza G, Bullitta S (2011) Correlations between phenolic content and antioxidant properties in twenty-four plant species of traditional ethnoveterinary use in the Mediterranean area. Pharm Biol 49(3): 240-247.

24. Nunes R, Carvalho IS (2013) Antioxidant activities, distribution of phenolics and free amino acids of Erica australis L. leaves and flowers collected in Algarve, Portugal. Nat Prod Res 27(18): 1664-1667. 